\title{
Tree-decorated planar maps
}

\author{
Luis Fredes* \\ LaBRI \\ Université de Bordeaux. \\ Bordeaux, France \\ luis-maximiliano.fredes-carrasco@u-bordeaux.fr
}

\author{
Avelio Sepúlveda ${ }^{\dagger}$ \\ Institut Camille Jordan \\ Univ Lyon, Université Claude Bernard Lyon 1, CNRS UMR 5208 \\ Villeurbanne, France \\ sepulveda@math.univ-lyon1.fr
}

Submitted: Apr 4, 2019; Accepted: Mar 7, 2020; Published: Mar 20, 2020

(C) The authors. Released under the CC BY-ND license (International 4.0).

\begin{abstract}
We introduce the set of (non-spanning) tree-decorated planar maps, and show that they are in bijection with the Cartesian product between the set of trees and the set of maps with a simple boundary. As a consequence, we count the number of tree decorated triangulations and quadrangulations with a given number of faces and for a given size of the tree. Finally, we generalise the bijection to study other types of decorated planar maps and obtain explicit counting formulas for them.
\end{abstract}

Mathematics Subject Classifications: 05A19,05C30,60D05

\section{Introduction}

In this paper, we study the combinatorial properties of tree-decorated maps via the use of a simple bijection. A tree-decorated map is a (planar) map given with a distinguished subset of the edges making up a tree. Tree-decorated maps form a one-parameter family interpolating between planar maps, when the subtree has 1 edge, and spanning-tree decorated maps, when the subtree has the same number of vertices as the whole map.

*Supported by ANR GRAAL (ANR-14-CE25-0014).

†Supported by ERC grant LiKo 676999 


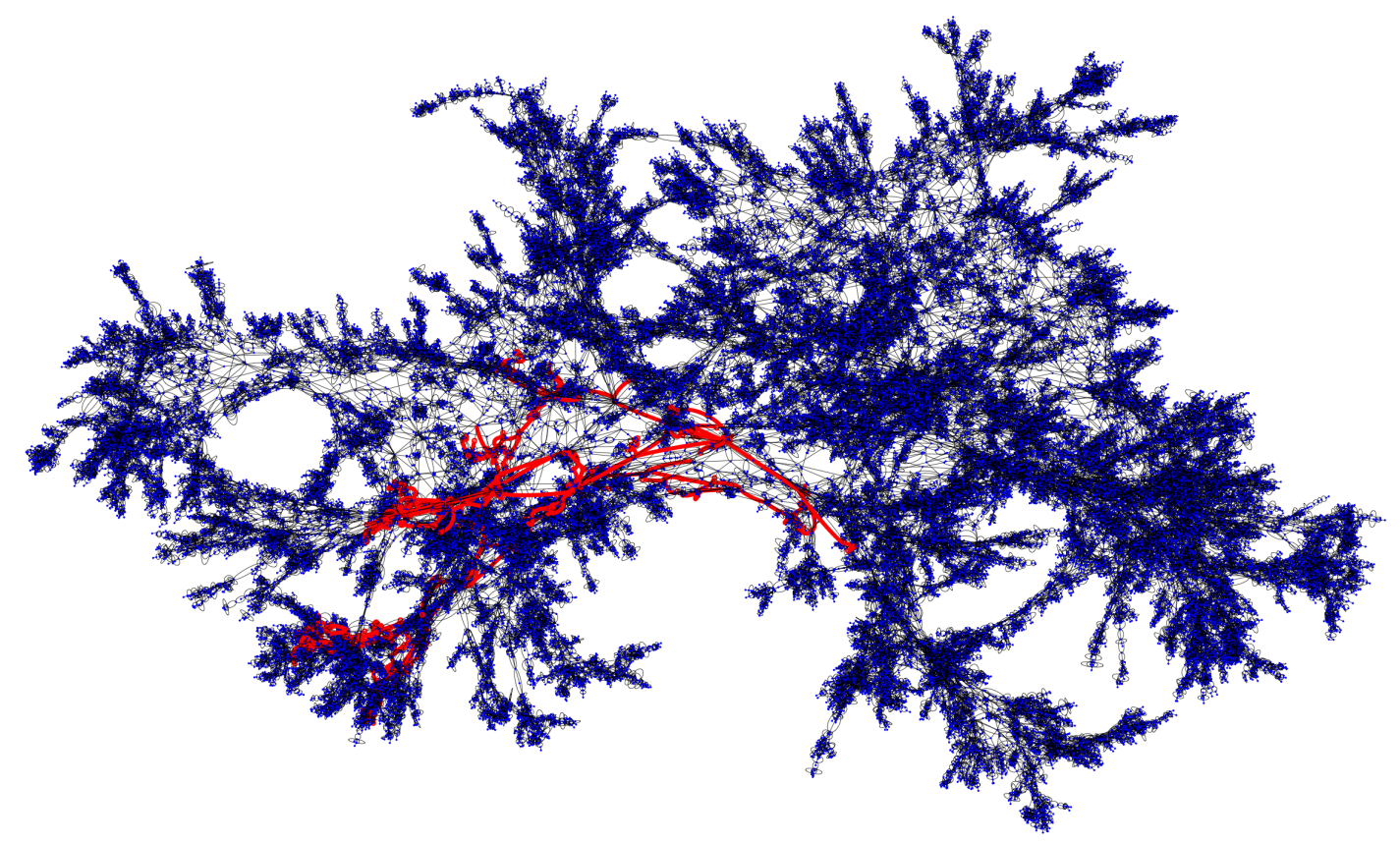

Figure 1: A simulation, based on the bijection introduced on this paper, of a uniformly chosen tree decorated quadrangulation with 90000 faces and with a tree of 500 edges. The tree is in red and the image is close to an isometrically embedding of the map in $\mathbb{R}^{3}$.

Planar maps were introduced in [Edm60] and afterwards have been thoroughly studied in many works from both a combinatorial (see for example [Tut62, Sch98, BDFG04]) and a probabilistic perspective (see for example [MM06, LG13, Mie13]). Spanning-tree decorated maps were first studied in [Mul67], where they obtain a simple closed formula to count them, which later was interpreted in [LW72, CDV86, Ber07] through bijective methods. These bijections became key to the study of planar maps decorated by statistical physics models [She16].

\subsection{Motivation}

The main motivation for the introduction of this model is to try to understand the difference, as metric spaces, between uniformly chosen planar maps and uniformly chosen spanning-tree decorated planar maps.

The study of planar maps as metric spaces has been an active area of research in probabilities these last years. The Cori-Vauquelin-Schaeffer bijection [Sch98] has been used to understand the case of uniformly chosen planar quadrangulation with $f$ faces. Some asymptotic properties are: the distances scale like the number of edges to the power $1 / 4$ and there is an explicit limiting metric space called Brownian map [MM06, Mie13, LG13]. However, in the world of uniformly chosen spanning-trees decorated planar maps, we do not know much. Only bounds on the order of the diameter as a function of the number of edges are known [GHS17, DG18]. On the optimistic side, Lehman and Walsh's 
bijection [LW72] shows that, as a plane tree, the spanning tree decorating the map is chosen uniformly over all trees with a given number of edges.

The main reason why it is difficult to understand distances in the context of spanningtree decorated maps becomes clear when one compares it to the case of planar quadrangulations. The main tool used to study distances in these planar maps is the (CVS) bijection, which relates a planar map to a pair of trees. In this bijection, one of the trees encodes the distance to a marked point, thus by knowing this tree, one gets an immediate upper bound on the diameter of the map. On the side of tree-decorated planar maps, we are not that lucky. Even though Lehman and Walsh's bijections [LW72, Ber07] relate them to a pair of trees, it is not possible to extract any information about distances in the original graph from just looking at either of them.

At this point, let us make a remark from the point of view of conformal field theory: uniformly chosen planar maps are not expected to have the same scaling limit as the spanning-tree decorated planar maps. This is because, while uniformly chosen planar maps are models with central charge equal to $0(c=0)$, spanning-tree decorated maps have an associated central charge of $-2(c=-2)$ [JS04]. Recall that tree-decorated planar maps interpolate between these two models. Trying to understand how this interpolation works in the limit is the main interest of the follow-up paper [FS19].

\subsection{Results}

Let us, now, present the main results of the paper. For detailed definitions, we refer the reader to Section 2.

The main result of this paper is an explicit bijection between tree-decorated maps and pairs consisting in a map with a simple boundary and a planar tree.

Theorem 1. The set of tree-decorated maps with $f$ faces decorated by a tree with $m$ edges and a root-edge on the tree, is in bijection with the Cartesian product of the set of trees with $m$ edges and the set of maps with a simple boundary of size $2 m$ with $f$ interior faces.

The bijection can be summarise as follows: the tree is kept and the map with a boundary is produced by a duplication of the edges of the tree. We call this direction of the bijection ungluing, and its inverse gluing. Note that the gluing consists only in identifying the boundary of the map using the equivalence relationship generated by the tree. Additionally, let us note that as the decorating tree is kept without any changes, in a uniformly chosen tree-decorated map, the law of the tree is uniform among all plane trees of a given size (Corollary 13). This is a generalisation of the result for spanningtrees decorated maps. Furthermore, this also shows that the bijection also works when restricted to maps decorated by a special type of tree, e.g. binary trees, etc..

The main contribution of Theorem 1 is to connect the study of tree-decorated maps to that of maps with a boundary. In the spirit of the motivation, note that maps with boundary are also an interpolation model, as when the boundary is of size 2 , we recover planar maps, and, when the boundary has twice the number of edges, we recover planar trees. This effect was explored, for uniformly chosen maps with a boundary in [Bet15], 
and we hope that the bijection allows us to transfer the transition from map to a tree to that from a map to a spanning tree-decorated map.

Important consequences of Theorem 1 are counting formulas for some subsets of treedecorated maps. A close look at the bijection of Theorem 1 shows that the ungluing procedure changes only the newly created face. Thus, it is possible to obtain counting formulas for tree-decorated (and spanning-tree decorated) maps with prescribed face degree distribution, e.g. $q$-angulations. To obtain these results, we need to count maps with a simple boundary and use a re-rooting argument. Luckily, we can find these countings in [Kri07] for triangulations and in [BG09] for quadrangulations.

Corollary 2. For $m \leqslant f / 2+1$, the number of tree-decorated triangulations where the tree has $m$ edges and the map has $f$ faces is

$$
2^{f-2 m} \frac{(3 f / 2+m-2) ! !}{(f / 2-m+1) !(f / 2+3 m) ! !} \frac{3 f}{m+1}\left(\begin{array}{c}
4 m \\
2 m, m, m
\end{array}\right),
$$

where $n$ !! stands for the double factorial of $n$.

Furthermore, for $m \leqslant f+1$, the number of tree-decorated quadrangulations, where the tree has size $m$ and the map has $f$ faces is

$$
3^{f-m} \frac{(2 f+m-1) !}{(f+2 m) !(f-m+1) !} \frac{4 f}{m+1}\left(\begin{array}{c}
3 m \\
m, m, m
\end{array}\right) .
$$

Note that triangulations, resp. quadrangulations, with $f$ faces have $f / 2+2$ vertices, resp. $f+2$ vertices. Thus, one can also find the number of spanning tree-decorated triangulations and quadrangulations. These formulas appeared first in [BM11], and were based in Lehman and Walsh's bijection [LW72]. Thus, these results give a new bijective proof of them.

Corollary 3. The number of spanning-tree decorated triangulations with $f$ faces, where the root-edge may be placed in every possible oriented edge (not necessarily in the spanningtree) is

$$
\frac{12 f}{(f+4)(f+2)^{2}}\left(\begin{array}{c}
2 f \\
f, f / 2, f / 2
\end{array}\right) \text {. }
$$

Furthermore, the cardinal of the set of spanning-tree decorated quadrangulation with $f$ faces where the root-edge may be placed in every possible oriented edge (not necessarily in the spanning-tree) is

$$
\frac{4 f}{(f+1)^{2}(f+2)}\left(\begin{array}{c}
3 f \\
f, f, f
\end{array}\right) \text {. }
$$

Let us remark that the counting formula for general maps with given boundary length and number of edges exists and is not closed so we decided not to present it here. Nevertheless, we compute its generating function in Section 4.2. Furthermore, note that the spanning-tree decorated maps with a given number of faces is infinite, so as it is stated the bijection is not interesting in that case. Nevertheless, given that the bijection does not change the vertices, edges and faces in the interior the same proof can be adapted to obtain the following result. 
Theorem 4. The set of tree-decorated maps with $e+m$ edges decorated by a tree with $m$ edges and a root-edge on the tree, is in bijection with the Cartesian product of trees with $m$ edges and maps with a simple boundary of size $2 m$ and $e$ interior edges.

The main bijection is not only useful to obtain counting results, but we plan to use it in [FS19] to study distances in uniformly chosen tree-decorated maps. The bijection becomes useful because distances in uniformly chosen maps with boundaries (simple or not) have been intensively studied [Bet15, BM17, GM19] and are better understood than that of tree-decorated maps.

Let us, also, mention that the bijection presented in this work is simple enough so that decoration on the vertices can be carried between the two objects. We believe that this fact may be an important tool to understand the behaviour of random tree-decorated maps whose underlying distribution is not uniform, but proportional to the partition function of a statistical physic model. These probability laws have been object of great interest in the statistical physics community, especially after the introduction of the so-called 'Hamburger-Cheeseburger'-bijection [She16].

Most of the other results of this article consist in using the main idea of the bijection of Theorem 1 to produce bijections between other combinatorial objects. In this flavour, we study forest-decorated planar maps (see Corollary 21) and tree-decorated planar maps with a simple boundary (see Corollary 20). The latter one allows us to make the gluing procedure in a progressive dynamical way (see Proposition 16).

On another note, we also explain what happens when one tries to use our gluing procedure with maps whose boundary is not necessarily simple. In this case, the gluing does not produce maps, but what we call bubble-maps (see Section 3.3), which are graphs embedded in a tree-like structure of spheres, and which are decorated by a specific type of circuit (see Proposition 16).

As a final remark, we would like to say that gluing maps along the boundary has already been done, but not to study the case where the decoration is a uniform tree. Some gluings in the literature are, for example, self avoiding walks [DK88, Bet15, GM16, CC16], loop erased-random walks [MS16] and loops [BBG11].

\subsection{Organisation of the paper}

The paper is organised as follows: we start with the preliminaries, where we carefully present the classical objects that are important for the paper together with the maps we are interested in. In Section 3, we present all the bijections and their proofs. Finally, in Section 4, we discuss the counting formulas we obtain from the bijections.

\section{Preliminaries}

\subsection{Elementary definitions}

In this section, we present the elementary concepts that appear in this work. As the main result of the paper is a self-contained bijection, the counting results are only stated 
when needed. For an introduction to planar maps, we recommend, for example, to see [GJ04, FS09] and [BM11].

A ROOTED PLANAR MAP, or MAP for short, is a finite connected graph embedded in the sphere that has a marked oriented edge. We consider that two maps are the same if there is an homeomorphism between them that preserves the orientation (i.e. respecting a cyclic order around every vertex). We call ROOT EDGE the marked oriented edge and ROOT VERTEX its starting point.

The FACES of a map are the connected components of the complement of the edges in the embedding. The DEGREE of a face is the number of oriented edges for which the face lies at its left. A $q$-ANGULATiOn is a map where each of the faces has degree $q$. We set $\mathrm{M}_{f}$ to be the set of all maps with $f$ faces. Finally, we call a PLANTED PLANE TREE, or TREE for short, a map with a single face and we denote the set of planted plane trees with $m$ edges by $\mathrm{T}_{m}$.

We will encode trees of $T_{m}$ using walks. In the literature, one can find several of these codings, see for example Section 1 of [LG05]. Here we are interested in the contour function. This is a bijection that associates to each rooted plane tree with $m$ edges a Dyck path $C$ indexed by $\llbracket 0,2 m \rrbracket$. For a more detailed description we refer to Section 1.1 of [LG05] and Section 2 of [Ber07].

A rooted plane tree $\mathfrak{t}$ has an intrinsic way of visiting all of its oriented-edges. This visit can be represented by a walker that starts from the root vertex and turns around the tree. The walker follows the direction of the root edge touching the tree with his left hand $^{1}$ as long as it walks. The walker, then, continues until he returns to the root edge. Note that this walk visits every oriented edge only once. Now, we define the contour function of $\mathfrak{t} C_{\mathfrak{t}}: \llbracket 0,2 m \rrbracket \rightarrow \mathbb{N}$ as the function for which $C_{\mathfrak{t}}(n)$ is the distance to the root vertex (height) of the vertex visited at time $n$ by the walker (time 0 for the root vertex).

The inverse of this bijection $\mathfrak{t} \rightarrow C_{\mathfrak{t}}$ is explicit. We say that a function $C: \llbracket 0,2 m \rrbracket \rightarrow \mathbb{N}$ is a CONTOUR FUnCTION if $C(0)=C(2 m)=0$ and its increments are \pm 1 , i.e., $C$ is a Dyck path. We can construct a plane tree from a contour function by saying that two points $n_{1}, n_{2} \in \llbracket 0,2 m \rrbracket$ are equivalent if for $n_{1} \leqslant n_{2}$

$$
C\left(n_{1}\right)=C\left(n_{2}\right)=\inf _{n \in \llbracket n_{1}, n_{2} \rrbracket} C(n) .
$$

The vertices of the tree are the equivalence classes of the relation and the edges can be recovered as follows: two vertices have an edge between them if there exists one element in each of their equivalent classes that are exactly at distance 1 in $\llbracket 0,2 m \rrbracket$. Note that each edge is associated with exactly two steps of the walk, one going up and one going down.

We define a NON-SELF CROSSING CIRCUIT as a sequence of directed edges $\left(e_{i}\right)_{i=0}^{l-1}$, for some $l \in \mathbb{N}$, embedded in the plane, such that the head of $e_{i}$ is the tail of $e_{i+1} \bmod l$ for all $i \in \llbracket 0, l-1 \rrbracket$, and that it does not cross itself. The last part means that for every vertex in the circuit, we do not find the pattern $e_{i}, e_{j+1}, e_{i+1}, e_{j}$ in cyclical order, where $i, j \in \llbracket 0, l-1 \rrbracket$ and the sum in the indexes is modulo $l$.

\footnotetext{
${ }^{1}$ Note that, in the literature, the walker usually walks following its right hand. In this work, the left hand convention simplify some statements.
} 


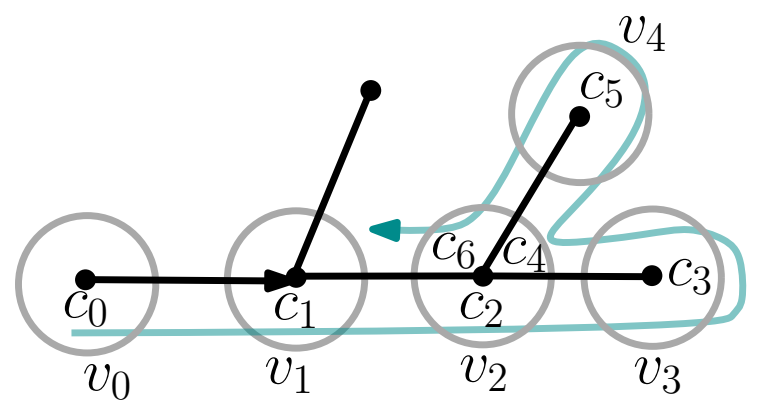

Figure 2: Tree with part of the contour in green, the corners are numbered as $c_{i}$ and are shown in gray. All the corners belonging to the same circle belong to the same equivalence class associated with a vertex, for example $v_{2}=\left[c_{2}\right]_{c}=\left[c_{4}\right]_{c}=\left[c_{6}\right]_{c}$.

The face that is at the left of the root edge will be called the ROOT FACE. In what follows, MAPS WITH A BOUNDARY are maps where the root face plays a special role. The set of oriented edges that have the root face to its left are called the BOUNDARY. The number of oriented edges in the boundary will be called its size. The boundary will be seen either as a set of oriented-edges or as a cyclic path around the root face, modulo cyclic rotation of the indices. All faces different from the root face are called INTERNAL FACES.

We denote by $\mathrm{B}_{f, m}$ the set of maps with boundary of size $m$ with $f$ internal faces. Note that each oriented edges in the boundary of a map has a canonical label in $\llbracket 0, m-1 \rrbracket:=$ $\{0,1, \ldots, m-1\}$ coming from the number of steps that a walker, who starts from the root edge and who follows the boundary of the root face, takes to arrive to a given edge (label 0 for the root edge).

When the boundary of the map is SIMPLE, i.e., the boundary is not vertex-intersecting, the labels of the edges induce a labeling of the vertices of the boundary (the label of the root-vertex is 0 ). The set of maps with a simple boundary in $\mathrm{B}_{f, m}$ is denoted by $\mathrm{SB}_{f, m}$.

Let us also introduce another type of boundary. A boundary is called BRIDGELESS if the walk described above never goes twice along the same edge. Let us give an alternative definition. An edge is called a bridge if its suppression disconnects the map. Thus, a boundary is said to be bridgeless if it does not contain any bridges.

A map $\mathfrak{m}_{1}$ is said to be a submap of $\mathfrak{m}_{2}$ (with the notation $\mathfrak{m}_{1} \subset_{M} \mathfrak{m}_{2}$ ) if $\mathfrak{m}_{1}$ can be obtained from $\mathfrak{m}_{2}$ by suppressing edges and vertices. This definition implies that $\mathfrak{m}_{1}$ respects the cyclic order of $\mathfrak{m}_{2}$ in the vertices and edges remaining. A decorated map is a map with a special submap, i.e. a pair $(\mathfrak{m}, \mathfrak{s m}(\mathfrak{m}))$ with $\mathfrak{m} \in \mathbf{M}_{f}$ for some $f \in \mathbb{N}$ and $\mathfrak{s m}(\mathfrak{m}) \subset_{M} \mathfrak{m}$.

\subsection{Objects of interest and notation}

We present, now, the main objects of study of the paper. In particular, we rigorously define tree-decorated maps.

Take a map $\mathfrak{m} \in \mathrm{M}_{f}$ and define $\mathrm{T}_{m}(\mathfrak{m})$ to be the set of (unrooted) trees $\mathfrak{t} \subset_{M} \mathfrak{m}$ with $m$ edges. A TREE-DECORATED MAP is a pair $(\mathfrak{m}, \mathfrak{t})$ with $\mathfrak{m} \in \mathbf{M}_{f}$ and $\mathfrak{t} \in \mathrm{T}_{m}(\mathfrak{m})$. 
We denote by $\mathrm{M}_{f}^{\top, m}$ the set of all of these pairs. Furthermore, we denote $\stackrel{\mathrm{M}}{f}^{\top, m}$ the set of all tree-decorated maps where the root of the map is in the tree. Note that one can do similar definitions when the map $\mathfrak{m}$ is a $q$-angulation. The set of tree-decorated triangulations and quadrangulations is counted in Corollary 2 and that of spanning-tree decorated triangulations and quadrangulations is counted in Corollary 3.

Let us now consider a map with a simple boundary $\mathfrak{m}^{b} \in \mathrm{SB}_{f, m_{1}}$ and define $\mathrm{T}_{m_{2}}^{b}(\mathfrak{m})$ to be the set of trees $\mathfrak{t} \subset_{M} \mathfrak{m}^{b}$ with $m_{2}$ edges containing a unique vertex on the boundary, the root vertex. A TREE-DECORATED MAP WITH A SIMPLE BOUNDARY is a pair $\left(\mathfrak{m}^{b}, \mathfrak{t}\right)$ with $\mathfrak{m}^{b} \in \mathrm{SB}_{f, m_{1}}$ and $\mathfrak{t} \in \mathrm{T}_{m_{2}}^{b}\left(\mathfrak{m}^{b}\right)$. We define the set of all these pairs as $\mathrm{B}_{f, m_{1}}^{\mathrm{T}, m_{2}}$. The set of all tree-decorated triangulations with a simple boundary is counted by (16) and that of quadrangulations is counted by (17).

\section{Main Bijections}

\subsection{The basic bijection}

\subsubsection{From tree decorated maps to maps with simple boundary and trees}

In the following paragraphs, we construct the first part of the bijection: the ungluing procedure. This procedure is represented by a function $\phi$ that takes a tree-decorated map in $\stackrel{\circ}{\mathrm{M}}_{f}^{\mathrm{T}, m}$ and gives a tree in $\mathrm{T}_{m}$ together with a map with a simple boundary in $\mathrm{SB}_{f, 2 m}$. Basically, in this procedure, the resulting tree is equal to the decorating tree and the map with a boundary is obtained by a duplication of the oriented edges of the tree in such a way that the newly appeared oriented edges form a face, see Figure 3.
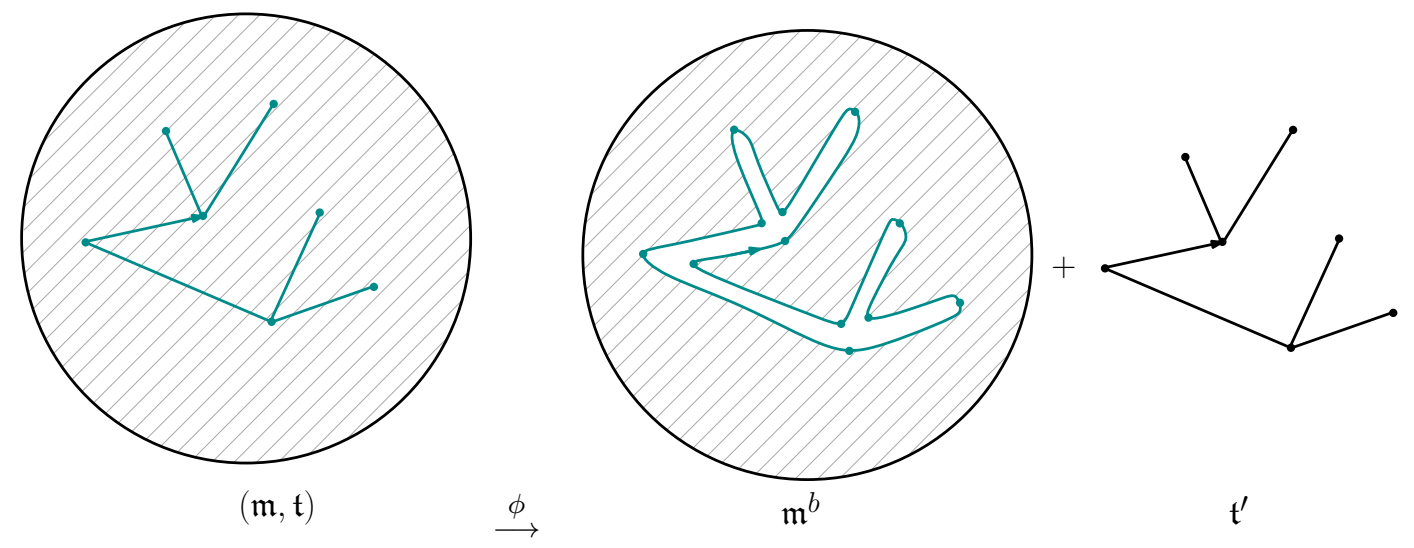

Figure 3: A simple sketch of the ungluing procedure.

Consider a tree decorated map $(\mathfrak{m}, \mathfrak{t}) \in \stackrel{\circ}{M}_{f}^{\top}, m$, and denote $\left(\mathfrak{m}^{b}, \mathfrak{t}^{\prime}\right)$ as the (soon-to-beconstructed) image of $(\mathfrak{m}, \mathfrak{t})$ under $\phi$. The tree $\mathfrak{t}^{\prime}$ is taken equal to the tree $\mathfrak{t}$, in particular that the root edge of $\mathfrak{t}^{\prime}$ is the same as that of $\mathfrak{t}$, i.e., the one of the tree decorated map.

To construct the map with a simple boundary $\mathfrak{m}^{b}$, we start defining the notion of a corner of $\mathfrak{t}$ at a vertex $x$ as a pair of two consecutive oriented edges (for the clockwise order), where the first one finishes at $x$ and the second one starts at $x$. Define $K$ as the 
set of corners of $\mathfrak{t}$. An oriented edge that starts or ends in $x$ is said to go to a corner $\left(e_{1}, e_{2}\right)$ of $x$ if it is between $e_{1}$ and $e_{2}$ for the clockwise order.

We can now define $\mathfrak{m}^{b}$. The set of vertices of $\mathfrak{m}^{b}$ is the union between the subset of vertices of $\mathfrak{m}$ that are not in the tree $\mathfrak{t}$, i.e. $V(\mathfrak{m}) \backslash V(\mathfrak{t})$, and $K$. To define the edges of $\mathfrak{m}^{b}$ we will use those of $\mathfrak{m}$. Each oriented edge $\vec{e}$ of $\mathfrak{m}$ whose vertices do not lie in $\mathfrak{t}$ is also an edge of $\mathfrak{m}^{b}$. Each oriented edge $\vec{e}$ of $\mathfrak{m}$ that has at least one vertex in $\mathfrak{t}$ becomes an oriented edge, that instead of being connected to the vertex in the tree, is connected to the corner in which the edge is incident. Finally, we add the oriented edges $\left(c_{1}, c_{2}\right)$ between two elements of $K$ if $c_{1}$ and $c_{2}$ seen as corners in $\mathfrak{t}$ are connected by an oriented edge in $\mathfrak{t}$.

To finish the definition of $\mathfrak{m}^{b}$ we are only missing the root edge and the embedding. The root edge of $\mathfrak{m}^{b}$ is the edge created from the root edge of $\mathfrak{m}$ by the procedure described above. The embedding is characterised by the cyclic orientation of the edges which is the same as that of $\mathfrak{m}$. In other words, in a corner the cyclic orientation is kept the same as in $\mathfrak{m}$, and this can be done because all edges in $\mathfrak{m}^{b}$ that belong to a corner can be identify with a unique edge in $\mathfrak{m}$.
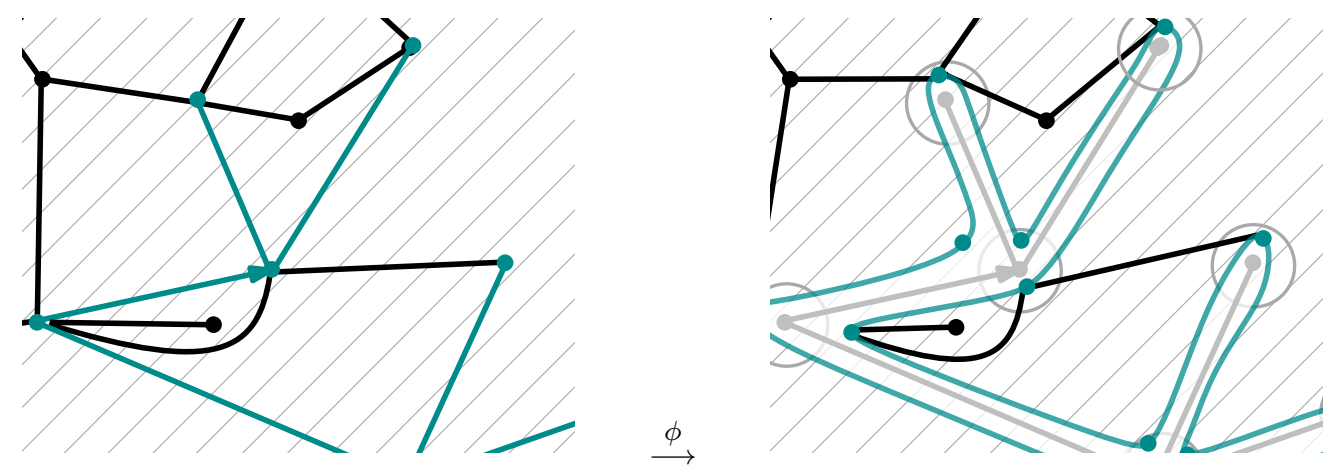

Figure 4: Local image of $\phi$

Let us, now, show the following result.

Lemma 5. The constructed map $\mathfrak{m}^{b}$ belongs to $\mathrm{SB}_{f, 2 m}$.

Proof. First, the boundary has clearly size $2 m$, since the edges of $\mathfrak{t}$ have been duplicated. Furthermore, the boundary of the map $\mathfrak{m}^{b}$ is simple, otherwise the tree would contain a cycle.

To finish, we just need to show that $\mathfrak{m}^{b}$ is a (planar) map, thus that it can be embedded in the sphere, $\mathbf{S}^{2}$. This comes directly from the fact that there is an orientation preserving isomorphism that goes from $\mathbf{S}^{2} \backslash \mathfrak{t}$ to $\mathbf{S}^{2} \cap\{(x, y, z): x>0\}$, and that can be extended to the boundary in a way that it sends prime ends of $\mathfrak{t}$ in $\mathbf{S}^{2} \backslash \mathfrak{t}$ to points in $\mathbf{S}^{2} \cap\{x=0\}$ keeping the cyclical order between them ${ }^{2}$. Thus, one can embed $\mathfrak{m}^{b}$ in $\mathbf{S}^{2}$ using any of

\footnotetext{
${ }^{2}$ In this case it follows from the fact that $\mathbf{S}^{2} \backslash \mathfrak{t}$ is simply connected (so one can use Riemman's theorem), together with results of the behaviour of conformal functions close to the boundary. See Chapter 2 of [Pom13] for definitions of prime ends and the main results in the boundary behaviour of conformal maps.
} 
these functions. To finish, it is enough to see that any of this isomorphisms produce an embedding of $\mathfrak{m}^{b}$ with boundary contained in $\mathbf{S}^{2} \cap\{x=0\}$.

\subsubsection{From maps with simple boundary and trees to tree decorated maps}

It is time to define the gluing function $\psi$ that takes as argument a map with a boundary in $\mathrm{SB}_{f, 2 m}$ and a tree in $\mathrm{T}_{m}$ and returns as result a tree-decorated map decorated in a tree of size $m$ and is the inverse of the ungluing function $\phi$. Informally, $\psi$ should identify two oriented edges incident to the external face of the map using the relation given by the oriented edges in the tree.

Let $\left(\mathfrak{m}^{b}, \mathfrak{t}^{\prime}\right)$ be an element of $\mathrm{SB}_{f, 2 m} \times \mathrm{T}_{m}$ and construct $(\mathfrak{m}, \mathfrak{t}) \in \stackrel{\circ}{ }_{f}^{\top, m}$, the value of $\psi\left(\left(\mathfrak{m}^{b}, \mathfrak{t}^{\prime}\right)\right)$ as follows. Recall that the vertices of the external face of $\mathfrak{m}^{b}$ are indexed from 0 to $2 m-1$, and call $C$ the contour function of $\mathfrak{t}^{\prime}$. Recall that $C$ induces an equivalent relation on vertices via equation (5), and define $V^{\prime}$ as the set of equivalence classes.

Let us now construct $\mathfrak{m}$. The vertex set is made by the union of $V^{\prime}$ with all vertices of $\mathfrak{m}^{b}$ that do not belong to the exterior face. The edge set of $\mathfrak{m}$ is constructed from those of $\mathfrak{m}^{b}$ in the following way. Let $(x, y)$ be an oriented edge of $\mathfrak{m}^{b}$, then the edge $(G(x), G(y))$ is in $\mathfrak{m}^{b}$, where

$$
G(x):= \begin{cases}{[l]} & \text { if } x \in V^{\prime} \\ x & \text { else, }\end{cases}
$$

where $l$ is the label of $x$ in the boundary, and $[l]$ is the equivalence class of $l$ under the equivalence relation defined by $C$.

Before defining the embedding, let us give some properties of the resulting graph.

- If $(x, y)$ is an oriented edge that belongs to the boundary of $\mathfrak{m}^{b}$, its reverse $(y, x)$ is associated exactly to one other edge $\left(y^{\prime}, x^{\prime}\right)$ such that $\left(x^{\prime}, y^{\prime}\right)$ belongs to the boundary of $\mathfrak{m}^{b}$. In other words, $C$ induces a perfect matching of the edges in the boundary

- As the boundary of $\mathfrak{m}^{b}$ is simple, the image of the edges in the boundary of $\mathfrak{m}^{b}$ has the same tree structure as $\mathfrak{t}^{\prime}$. We define $\mathfrak{t}$ as this image.

To finish the construction of $\mathfrak{m}$, we need to set the cyclical order of the edges around each vertex in the map. If $v$ is a vertex of $\mathfrak{m}$ that does not belong to $V^{\prime}$, we set the order or the edges surrounding it as the order of $\mathfrak{m}^{b}$. In the case where $v \in V^{\prime}$, we consider the order as the gluing of orders, following the corner identification around $v$ (see Figure (5)). Note that this also makes that the cycle order of $\mathfrak{t}$ is the same that the one of $\mathfrak{t}^{\prime}$.

To finish, let us actually prove that there exists an embedding that satisfies the given properties.

Lemma 6. $(\mathfrak{m}, \mathfrak{t})$ belongs to $\stackrel{\mathrm{M}}{f}^{\mathrm{T}, m}$

Proof. As $\mathfrak{t}$ is a subset of $\mathfrak{m}$, is a tree and has $m$ edges, we just need to prove that there exists an embedding that satisfies the order we imposed. This is showed as Lemma 5, because of the existence of a orientation preserving isomorphism that goes from $\mathbf{S}^{2} \cap$ $\{(x, y, z): x>0\}$ to $\mathbf{S}^{2} \backslash \mathfrak{t}$, and that takes any cyclically ordered prime ends of $t$ in $\mathbf{S}^{2} \backslash \mathfrak{t}$ to elements of $\mathbf{S}^{2} \cap\{x=0\}$ keeping the same cyclical order. 


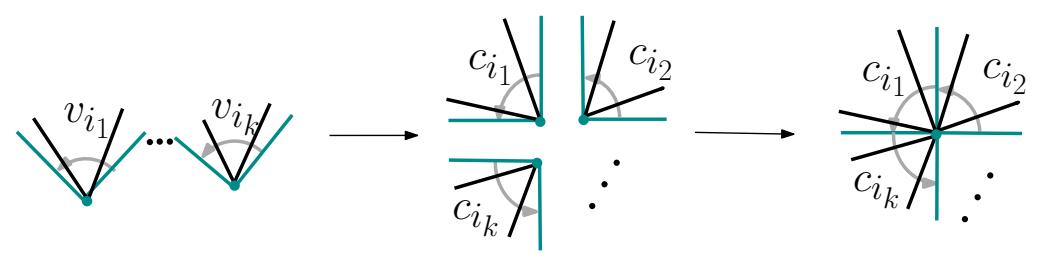

Figure 5: Gluing of corners and edge order around a vertex. For vertices $v_{i_{1}}, v_{i_{2}}, \ldots, v_{i_{k}}$ with corners in the same class, we associate their order of edges and we glue these orders following the corner sequence of the contour function.

\subsubsection{The gluing is actually a bijection}

Let us now prove that the gluing and ungluing are inverse of each other.

Proposition 7. For $f, m \in \mathbb{N}, \psi \circ \phi=I d$ in $\stackrel{\mathrm{M}}{f}^{\top}, m$ and $\phi \circ \psi=\operatorname{Id}$ in $\mathrm{SB}_{f, 2 m} \times \mathrm{T}_{m}$.

Proof. Consider $\mathfrak{m} \in \stackrel{\circ}{\mathbf{M}}_{f}^{\top}, m$, and let us show that $\psi(\phi(\mathfrak{m}))=\mathfrak{m}$. First note that the composition of both functions preserves the number of vertices of $\mathfrak{m}$. This is because, every vertex that does not belong to the decoration does not change by the transformations and the vertices on the decoration are separated by $\phi$ in corners and then gathered by $\psi$ in exactly the same vertices of $\mathfrak{m}$.

Let us now note that the edges are kept the same. This is because every edge without endpoints in the decoration remains after the transformations and edges with endpoints in the decoration again are unglued from the decoration by $\phi$ and glued after by $\psi$.

Finally, we see that the orientation of the edges is kept. The reason for this is that for vertices that are not in the tree the orientation is kept the same, and for edges that hit in the tree, the orientation is carried by the tree that is the same in both cases.

Remark 8. Proposition 7 can also be proven using that the isomorphisms taken in Lemma 5 and 6 may be chosen to be the inverse of each other.

Let us remark that the bijection $\phi$ makes the following correspondences.

\section{Tree-decorated map}

Faces of degree $q$

Vertices not in the tree of degree $d$

Edges not in the tree

Edges in the tree

Corners of the tree
(Map with a simple boundary, tree)

$\longleftrightarrow$ Internal faces of degree $q$

$\longleftrightarrow$ Internal vertices of degree $d$,

$\longleftrightarrow$ Internal edges,

$\longleftrightarrow$ Boundary edges,

$\longleftrightarrow$ Boundary vertices.

From the previous correspondences we conclude the following results.

Lemma 9. For any $q \in \mathbb{N}$, the ungluing function is a bijection between the set of treedecorated $q$-angulations with $f$ faces decorated by a tree of size $m$ and the Cartesian product between the set of q-angulation with simple boundary of size $2 m$ and the set of planar trees with $m$ edges. 
Lemma 10. The ungluing function is a bijection between the set of tree-decorated maps with $e+m$ total edges decorated by a tree of size $m$ and the Cartesian product between the set of maps with simple boundary of size $2 m$ with e internal edges and the set of planar trees with $m$ edges.

Remark 11. Let us also note that as vertex and faces are kept the same, except for the newly created external face, any face or vertex decoration commutes with the bijection.

Remark 12. The bijection can be restricted to different tree families. Since if $T \subset \mathrm{T}_{m}$, the gluing procedure produces a map decorated in a tree that belongs to $T$. Conversely, if $(\mathfrak{m}, \mathfrak{t})$ is a decorated map and $\mathfrak{t} \in T$, then the ungluing procedure gives a map with a boundary and $\mathfrak{t}^{\prime} \in T$.

Furthermore, as the ungluing keeps the tree without any changes we obtain the following probabilistic result.

Corollary 13. The tree of a uniformly chosen tree-decorated map with $f$ faces decorated by a tree of size $m$ is uniformly chosen in $\mathbf{T}_{m}$.

\subsection{Extensions}

In the following section, we discuss some extensions of the gluing procedure which allows us to make bijections between other combinatorial objects.

\subsubsection{Tree-decorated map with a simple boundary}

In this subsection, we discuss the gluing procedure between a tree and a map with a simple boundary that is bigger than the contour of the tree, and how this allows to create a dynamic gluing of the boundary.

For $f, m_{1}, m_{2} \in \mathbb{N}^{*}$, we extend the gluing function $\psi$ to $\mathrm{SB}_{f, m_{1}+2 m_{2}} \times \mathrm{T}_{m_{2}} \rightarrow \mathrm{B}_{f, m_{1}}^{\mathrm{T}, m_{2}}$, meaning that we glue a rooted tree with contour smaller than the boundary of a simple boundary map, the exterior face in this case shrinks but does not disappear. The resulting decorated map has a decoration that is a tree that shares only one vertex with the exterior face. We define the root edge of the resulting decorated map as the image of the edge labeled $2 m$ in the simple boundary map. This can be made formal, adapting the proof of theorem 1, to conclude the following proposition.

Proposition 14. Let $f, m_{1}, m_{2} \in \mathbb{N}$. The set of tree-decorated map with a simple boundary, $\mathrm{B}_{f, m_{1}}^{\mathrm{T}, m_{2}}$, is in bijection with the Cartesian product between trees and maps with a simple boundary, $\mathrm{T}_{m_{2}} \times \mathrm{SB}_{f, m_{1}+2 m_{2}}$.

Let us present a fact about gluing that we believe may become useful in the future, especially when working with local limits. This fact is going to be presented in probabilistic terms, as it may be its main use.

Take $x \in \llbracket 0, m-1 \rrbracket$ and a contour function $C$ of a tree of size $m$, i.e., a Dyck path. Then, for every level $l \in \mathbb{N}$ smaller than the value of $C(x)$ there is a unique connected maximal subset $\llbracket \underline{m}(x, l), \bar{m}(x, l) \rrbracket \ni x$ where $C(\cdot)-l$ is a Dyck path and

$$
l=C(\underline{m}(x, l))=C(\bar{m}(x, l)) .
$$


Note that the set of contour functions where this set is exactly $[\underline{m}, \bar{m}]$ can just be reconstructed by the Cartesian product between a positive path going from 0 to $l-1$ in $\underline{m}-1$ steps and a positive path going from $l-1$ to 0 in $\bar{m}-1$ steps.

Looking from a tree point of view, this decomposition allows us to progressively recover all the vertices of the tree whose common ancestors with $x$ is at distance at least $l$ from the root. This allows us to define a progressive procedure of gluing trees, where at time $t \in \llbracket 0, l-1 \rrbracket$ we glue the sub-tree whose vertices live in $\llbracket \underline{m}(x, l-t), \bar{m}(x, l-t) \rrbracket$ with a given map having a simple boundary of size $2 \mathrm{~m}$.

From a probabilistic perspective these remarks imply the following result.

Proposition 15. Let $\mathfrak{m}^{b}$ be a map with a boundary of size $2 m$ and $n$ vertices, chosen uniformly at random in $\mathrm{SB}_{f, m}$ and $\mathfrak{t}$ be a tree of size $m$, also chosen uniformly at random in $\mathrm{T}_{m}$. Additionally, take $x, \underline{m}, \bar{m} \in \mathbb{N}$ with $x \in \llbracket 0, m-1 \rrbracket$. Call $\mathfrak{m}_{t}$ the map generated by gluing the sub-tree generated by the vertices $\llbracket \underline{m}(x, l-t), \bar{m}(x, l-t) \rrbracket$ with $\mathfrak{m}^{b}$, where $l$ is the distance from $\mathfrak{t}$ to $x$. Conditionally on the event

$$
\{\llbracket \underline{m}(x, l-t), \bar{m}(x, l-t) \rrbracket=\llbracket \underline{m}, \bar{m} \rrbracket\},
$$

the law of $\mathfrak{m}_{t}$ is uniform over the $(\underline{m}-\bar{m}) / 2$-tree decorated maps with a simple boundary of size $m-(\underline{m}-\bar{m})$ and whose root is at cyclic distance $\min \{\underline{m}, m-\bar{m}\}$ of the tree. Now, call $\mathfrak{t}_{t}$ the tree decorating $\mathfrak{m}_{t}$. We have that under the above conditioning, the map $\mathfrak{m}_{t}$ minus $\mathfrak{t}_{t}$, the tree $\mathfrak{t}_{t}$ and the tree $\mathfrak{t}_{m} \backslash \mathfrak{t}_{t}$ are all independent.

In more probabilistic words, the maps $\mathfrak{m}_{t}$ works as a renewal submap for the gluing process of the tree.

\subsection{Gluing with a non-simple boundary}

To finish this section, we introduce a gluing procedure for the case when the boundary of the map glued is not simple. The combinatorial objects that appear do not seem canonical, so we discuss with more emphasis the complication that make them arise.

We mostly focus on the case, where the boundary is bridgeless. In that case, we can make sense of a generalization of the gluing procedure. After that, we will explain why the gluing procedure "does not make sense" when the boundary has bridges.

Let us start discussing the gluing between a map with a bridgeless boundary and a rooted tree. Note that, in this case, the resulting glued "map" has a decoration that is not necessarily a tree, but a submap (see Figure 6). This generates two problems.

The first one comes from the fact that the gluing is not injective. This is not a central problem as it can be fixed: instead of considering the decoration as a submap, one considers it as a non-self crossing circuit, defined in Section 2.1. The circuit is just the image of the contour walk of the tree under the gluing. The fact that the boundary was bridgeless, implies that the circuit only passes once by each oriented-edge.

The second problem that arises in the gluing is the main one: there are, in fact, two types of cycles that may appear in the circuit. To describe them, let us first note that cycles can only appear in vertices that come from a pinch point of the boundary. Thus, 
there are two possibilities for the image under the gluing of these vertices. Either, the gluing identifies the corners of a pinch point with different vertices of the tree. These generate cycles that preserve the topology.

On the other hand, if the gluing identifies two corners of a pinch point with the same vertex in the tree, then a "wicked" point appears. That is to say, this vertex pinches down the sphere, and the graph can no longer be embedded in the sphere but only in a topological space with bubbles.

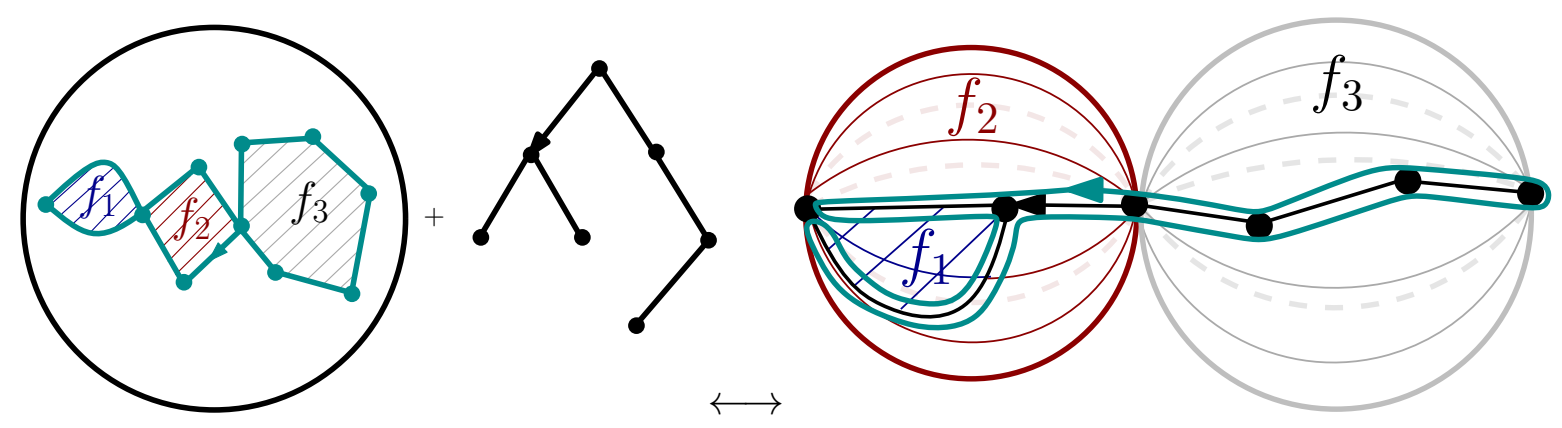

Figure 6: Left: Bridgeless map with a non-simple boundary (interior faces are filled) and a tree. Right: Bubbles (3D plot) formed by the gluing of a map with non-simple boundary and a tree. We chose to leave the scar generated by the tree after the gluing (black), even though the decoration is the green circuit (oriented edges following the direction of the root edge from the root edge).

Let us explain, in a better way, what happens with the wicked points. For that it is useful to assume that the map with a boundary is already embedded in the sphere. Let $x$ be a pinch point that generates a wicked point in the resulting object. We know that if we remove $x$, the map with a boundary is left with two or more connected components of interior faces, say $\mathfrak{C}_{1}$ and $\mathfrak{C}_{2}$. Note that two faces, $f_{1}$ belonging to $\mathfrak{C}_{1}$ and $f_{2}$ belonging to $\mathfrak{C}_{2}$ can only be connected through a continuous path in the sphere that either passes through $x$ or passes through the exterior face of the map. After the gluing has been done, the exterior face disappears. Thus, any continuous path that goes from the image of $f_{1}$ to that of $f_{2}$ has to pass by $x$, as the tree does not code the difference between these edges. Thus, the resulting glued "map", is not a map since it cannot be embedded in the sphere: removing the point $x$ results in a disconnection of the faces, which never happens in $\mathbf{S}^{2}$.

Additionally note that "bubbles" are connected in an arborescent way, as they do not form cycles. This is because, each bubble is associated with a starting point and a subtree of the original tree, where two subtrees can only intersect in at most one vertex (See Figure 6).

\subsubsection{Bubble maps definition}

In the next paragraphs, we will define more rigorously the notion of bubble maps. To do this, we need to define the "supporting topological spaces", which in contrast to the case of planar maps are surfaces that are not homeomorphic to $\mathbf{S}^{2}$. This is because the 
construction explained previously produces bubbles that can be imagined as on Figure 7 .

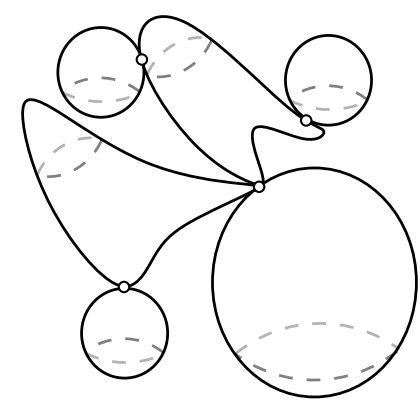

Figure 7: Bubble structure.

The "supporting topological spaces" that appear in the gluing are going to be called ORIENTED BUbBle tOPOLOGICAL SPACE (OBTS). They are are sets $S$ having the following characteristics:

- $S$ is the union of a finite number $b$ of surfaces, called thereafter "bubbles" $B_{1}, B_{2}, \ldots$, $B_{b}$ taken in a fixed order (meaning that the label $i$ of $B_{i}$ will play a role, see Figure 10 for an idea). The sequence of bubbles $\left(B_{1}, \cdots, B_{b}\right)$ satisfies:

- Each $B_{j}$ is oriented and homemorphic to $\mathbf{S}^{2}$.

- For every $j \neq j^{\prime}$ the intersection $B_{j} \cap B_{j^{\prime}}$ is either empty or a single point ${ }^{3}$.

- The bubble adjacency graph AG (to be defined) is a tree (see Figure 8).

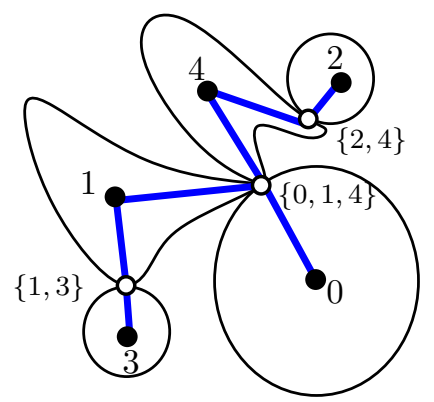

Figure 8: OBTS together with its adjacency graph in blue.

To finish the definition of an OBTS we need to define the adjacency graph. To do that we introduce the notion of a junction. We say that a set $A$ in $\operatorname{Pow}(\llbracket 1, b \rrbracket)$ (the powerset of the set $\llbracket 1, b \rrbracket)$ is a junction if

$$
\bigcap_{j \in A} B_{j} \neq \emptyset
$$

\footnotetext{
${ }^{3}$ We do not exclude the fact thant two bubbles may intersect at the same point.
} 

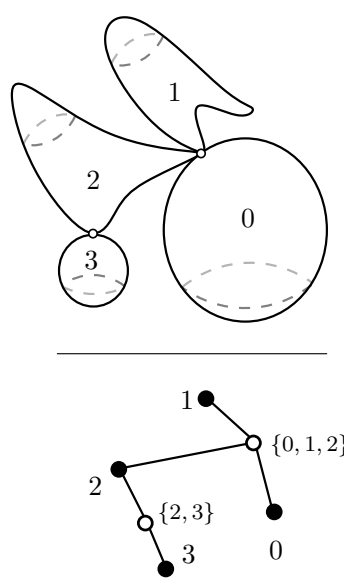

(a) An OBTS.

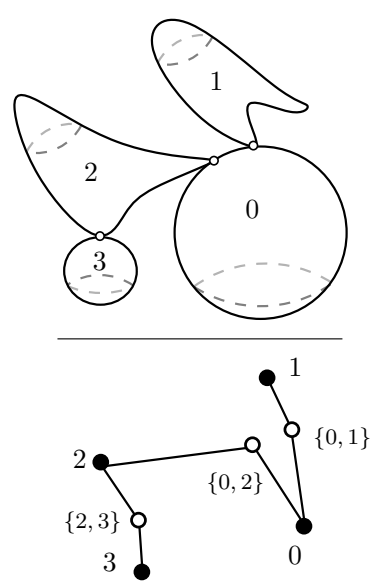

(b) An OBTS.

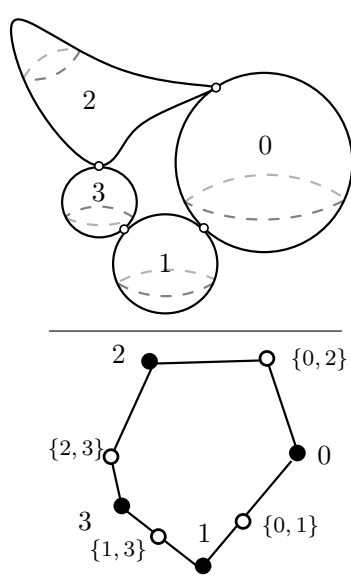

(c) Not an OBTS. AG

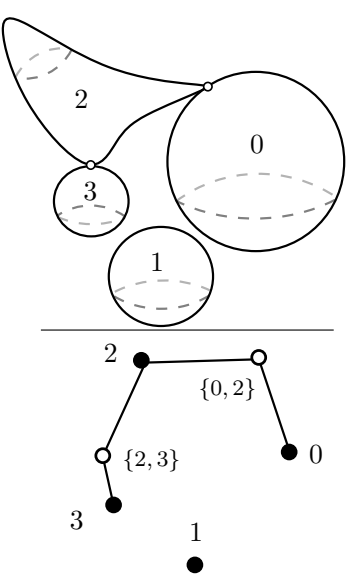

(d) Not an OBTS. AG

Figure 9: At the top sketch of different topologies. At the bottom the AG graph: white points are junctions while black vertices come from each bubble.

and if $A$ is maximal for the inclusion order. Each junction point records the labels of the bubbles that intersect at the same point. We call IP the set of all junction points. At this point it is important to notice that junctions and intersection points between bubbles are in correspondence. Now we define the graph AG by specifying its vertex and edge sets:

- The set of vertices is IP $\cup \llbracket 1, b \rrbracket$

- The edge set $E$ is the set of pairs $\{x, y\}$ where $x \in \mathbb{P}, y \in \llbracket 1, b \rrbracket$ and $y \in x$.

For an idea of OBTS's see Figure 9.

Now we consider a OBTS

$$
S=B_{1} \cup B_{2} \cup \cdots \cup B_{b} .
$$

as a "labeled" topological space (see Figure 10 for an intuition), meaning that it is more than a "formal union" of bubbles since each bubble comes with an orientation and rank in a list (to be totally formal, we should have written $\mathrm{S}$ as a function of the $b$-tupple $\left.\left(B_{1}, \ldots, B_{b}\right)\right)$.

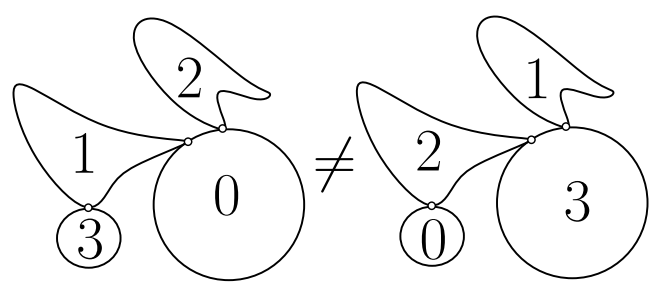

Figure 10: OBTS together with its adjacency graph in blue. 
We say that two OBTS $S=\cup_{i=1}^{b} B_{i}$ and $S^{\prime}=\cup_{i=1}^{b^{\prime}} B_{i}^{\prime}$ are equivalent if there exists an homeomorphism from $S$ to $S^{\prime}$ which preserves the labelings of bubbles and the orientation of bubbles in correspondence. We call such an homeomorphism GOOD HOMEOMORPHISM. Notice that $S$ equivalent to $S^{\prime}$ implies that $b=b^{\prime}$ and that the AG trees are the same as labeled graphs.

We call a PRE-BUBBLE MAP a pair $(D, S)$ where $S$ is an OBTS and $D$ is the drawing of a connected graph on $S$, such that for each junction point of $S$ there is a vertex point of $D$ and that the restriction of $D$ to each bubble is a proper cellular drawing (as usual for maps).

Finally, we call BUBBLE MAP the equivalence classes of pre-bubble maps for the following equivalence relation: $(D, S) \sim\left(D^{\prime}, S^{\prime}\right)$ if there exists $\phi$ a good homeomorphism $\phi: S \rightarrow S^{\prime}$, s.t. $\phi(D)=D^{\prime}$.

Returning to decorated bubble maps, let us note that the image of the path given by the walker around the tree, generates a non-self crossing circuit that passes exactly twice by each edge, more precisely, once per each oriented edge. From this circuit, it is possible to recover the original tree structure. Let us describe an algorithm that allows to recover the contour function $C$ of the tree by using the circuit. Start at $n=0$ and $C(0)=0$ from the root-vertex and following the root-edge, and iterate for each new edge of the circuit:

- If the edge has been visited, set $C(n+1)=C(n)-1$.

- If this is the first time the edge has been visited and it visits a new vertex, set $C(n+1)=C(n)+1$.

- If this is the first time the edge has been visited and it visits an already visited vertex, set $C(n+1)=C(n)+1$ and create a new non-self crossing circuit, where the visited vertex is duplicated. All edges visited before time $n$ (not including the one in this step), goes with the vertex going to the right, and all the others go with the vertex going to the left. At distance $\varepsilon>0$ from this point, the graph is embedded homeomorphically as before. This can be done because the circuit is not self-crossing.

- Set $n=n+1$.

Let us, now, define the image set of the gluing of a tree with a bridgeless map with a boundary. For $f, m \in \mathbb{N}$, we say that $(\mathfrak{m}, \mathfrak{c}) \in \stackrel{\mathrm{M}}{f}_{\text {, } \boldsymbol{m}}$ if: $\mathfrak{m}$ is a bubble-map with $f$ faces, $\mathfrak{c}$ is a non-crossing circuit with length $2 m$, going trough each edge twice, passing by every pinched point of the bubble-map and containing the root edge. Let us also define $\stackrel{\circ}{E}_{E=e}^{C, m}$ as $\stackrel{\mathrm{M}}{\mathrm{C}}_{f}^{\mathrm{C}, \mathrm{m}}$, where in the place of $f$ faces, we consider $e+m$ edges.

Let us summarize the discussion.

Proposition 16. When one glues a tree $\mathfrak{t}^{\prime} \in \mathrm{T}_{m}$ with a map with a bridgeless boundary $\mathfrak{m}^{b} \in \mathrm{B}_{f, 2 m}$, one obtains a map if and only if there is no vertex where the boundary of $\mathfrak{m}^{b}$ is not simple and that is identified by $\mathfrak{t}^{\prime}$.

More importantly, the gluing is a bijection between $\mathrm{T}_{m} \times \mathrm{B}_{f, 2 m}$ and $\mathrm{M}_{f}^{\mathrm{C}, \mathrm{m}}$. 
Proof. Given the above discussion, we only need to show that we can perform the ungluing. Let us note that the tree can be recovered from the algorithm described above. To recover the map with a boundary, one just needs to duplicate the edges crossed as described in Figure 4. Now, we only have to explain what needs to be done close to the pinch points. In those points, one just needs to locally modify the underlying space so that pinch points give rise to circular sectors and all these circular sectors belong to the internal face. The circular sectors are determined by the circuit.

Remark 17. Again, the bijection does not modify the degree of interior faces, so Proposition 16 is valid when we restrict our attention to bubble $q$-angulations.

It can be adapted to families of maps with a given number of edges.

Proposition 18. The set $\stackrel{\mathrm{M}}{E=e}_{E, \mathrm{~m}}^{\mathrm{s}}$ is in bijection with the Cartesian product of $\mathrm{T}_{m}$ with the set of maps with bridgeless boundary with e internal edges and boundary of size $2 \mathrm{~m}$.

Let us finish this section by describing the effect of a boundary with bridges, which prevents the extension of $\psi$. In this case, we do not only have problems with identification of vertices, but also with the identification of edges. In particular, there may be two bridges in the boundary having oriented edges identified in the tree. This makes that the circuit we create passes at least twice by the image of that oriented edge, and thus, it makes it impossible to reverse the gluing.
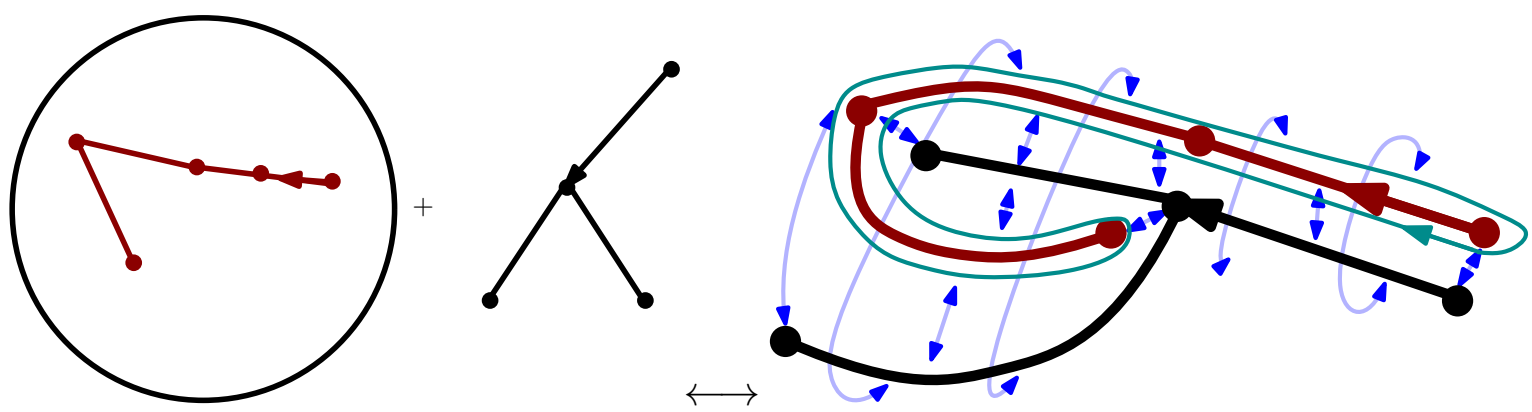

Figure 11: Left: Bridge map with a non-simple boundary (red tree) and a tree to glue.

Right: The gluing of the objects on the left. As in fig. 6 the green part represents the circuit (each edge in the red tree appears twice as we follow the root edge on the boundary of the root-face). We put blue lines for identifications made by the gluing. The edges of the black tree that do not contain the root-edge generate only one edge $e_{1}$, after the gluing, what makes the green circuit visit four times $e_{1}$.

\section{Countings}

In this section, we discuss how the bijections presented are translated in to counting formulas. Before stating the results, we need to introduce a re-rooting procedure, which allows us to obtain formulas for decorated maps whose root is not necessarily in the decoration. 
For convenience, in this section, for any set of maps $S$, we denote by $S(q)$ the subset of $S$ containing the maps whose faces (inner faces in the case of maps with boundary) have all degree $q$.

\subsection{Re-rooting procedure}

In this section, we explain what we call the re-rooting procedure. The motivation behind this comes from the fact that the decorated objects considered here so far have the rootedge in the decoration, while, for example in the literature, spanning-tree decorated maps have theirs root-edge in every possible oriented edge of the map. This procedure is quite general, instead we chose to explain the key idea on a particular family of objects.

Fix $r \geqslant 1$. A forest is a tuple of trees. We call $\mathfrak{f}$ an $r$-FOREST, if $\mathfrak{f}=\left(\mathfrak{t}_{i}, \vec{e}_{i}\right)_{i=1}^{r}$, where $\left(\mathfrak{t}_{i}, \vec{e}_{i}\right)$ is a rooted tree for all $i \in\{1,2, \ldots, r\}$. We define $\mathrm{F}_{m_{1}, m_{2}, \ldots, m_{r}}$ as the set of all $r$-forests such that for every $i \in\{1,2, \ldots, r\}, \mathfrak{t}_{i}$ has $m_{i}$ edges.

Define an $r$-BOUNDARIES MAP $\left(\mathfrak{m}, \vec{e}_{1}, \vec{e}_{2}, \ldots, \vec{e}_{r}\right)$ as a rooted map $\left(\mathfrak{m}, \vec{e}_{1}\right)$ and $\left(\vec{e}_{i}\right)_{i=1}^{r} r$ non intersecting edges of $\mathfrak{m}$. The map has multiple special (ordered) faces $f_{1}, f_{2}, \ldots, f_{r}$, called the boundary faces, where $f_{i}$ is the face to the left of $\vec{e}_{i}$; it is also required that the faces $f_{i}$ are simple and pairwise vertex-disjoint. We say that $\vec{e}_{i}$ is the root-edge of the $i$-th boundary. We denote by $\mathrm{MSB}_{f, m_{1}, \ldots, m_{r}}$ the set of all maps with $r$-boundaries (ordered), $f$ internal faces and with boundaries of size $m_{1}, \ldots, m_{r}$.

We also define a MULTIPLY ROOTED $r$-FOREST-DECORATED MAP as a decorated map $\left(\mathfrak{m},\left(\mathfrak{t}_{i}, \vec{e}_{i}\right)_{i=1}^{r}\right)$, where $\left(\mathfrak{m}, \vec{e}_{1}\right)$ is a rooted map and $\left(\mathfrak{t}_{i}, \vec{e}_{i}\right)_{i=1}^{r}$ is a forest with non vertexintersecting rooted trees with $\mathfrak{t}_{i} \subset_{M} \mathfrak{m}$. Notice that from this definition the root-edge of $\mathfrak{t}_{1}$ coincides with the root-edge of $\mathfrak{m}$. We define $\dot{M}_{f}^{\mathrm{F}, m_{1}, m_{2}, \ldots, m_{r}}$ as the set of multiply rooted $r$-forest-decorated maps where the size of $\mathfrak{t}_{i}$ is $m_{i}$ for all $i \in\{1,2, \ldots, r\}$.

The "re-rooting procedure" is needed in order to count multiply rooted $r$-forestdecorated map, when instead of considering $r$ root edges one considers just one that could be placed in every possible oriented edge of the map. For this, let us finally define a $r$-FOREST-DECORATED MAP as the decorated map $\left((\mathfrak{m}, \vec{e}),\left\{\mathfrak{t}_{i}\right\}_{i \in I}\right)$, where $(\mathfrak{m}, \vec{e})$ is a rooted map, $|I|=r$ and $\left\{\mathfrak{t}_{i}\right\}_{i \in I}$ is a set of non vertex-intersecting unrooted trees with $t_{i} \subset_{M} \mathfrak{m}$. Notice that we define the decorating forest $\left\{\mathfrak{t}_{i}\right\}_{i \in I}$ as a set. Consider an infinite vector $\vec{v}=\left(v_{1}, v_{2}, v_{3}, \ldots\right)$ with finitely many non-zero coordinates. We define the set $\mathbf{M}_{f}^{\mathrm{F}} \vec{v}$ of $r$-forest-decorated maps, for which there are among $\left\{\mathfrak{t}_{i}\right\}_{i \in I}$, for all $j \in \mathbb{N}^{*}, v_{j}$ trees with $j$ edges.

The main result of this section is the following formula

$$
\left|\stackrel{\mathrm{M}}{f}_{f}^{\mathrm{F}, m_{1}, . ., m_{r}}(q)\right| 2 m(f, q)=\left|\mathrm{M}_{f}^{\mathrm{F}, \vec{c}}(q)\right|\left(\prod_{i=1}^{r} 2 m_{i}\right) \prod_{k} c_{k} !
$$

where $m(f, q)$ is the number of edges of a $q$-angulation with $f$ faces, and where $\vec{c}$ is the infinite vector which encodes the multiplicity of the values $m_{i}$, i.e.

$$
c_{k}:=\left|\left\{i \in \llbracket 1, r \rrbracket: m_{i}=k\right\}\right|, \quad k \geqslant 1 .
$$


Let us now prove Equation (9). It just follows from counting a slightly bigger set. Let us call

$$
\begin{aligned}
& \tilde{\mathrm{M}}_{f}^{\mathrm{F}, m_{1}, . ., m_{r}}(q) \\
& :=\left\{\left(\mathfrak{m}, \vec{e},\left(\mathfrak{t}_{i}, \vec{e}_{i}\right)_{i=1}^{r}\right):\left(\mathfrak{m},\left(\mathfrak{t}_{i}, \vec{e}_{i}\right)_{i=1}^{r}\right) \in \dot{\mathrm{M}}_{f}^{\mathrm{F}, m_{1}, . ., m_{r}}(q) \text { and } \vec{e} \text { is an oriented edge of } \mathfrak{m}\right\} .
\end{aligned}
$$

In other words, an element of $\tilde{\mathrm{M}}_{f}^{\mathrm{F}, m_{1}, . ., m_{r}}(q)$ is made of an element of $\stackrel{\mathrm{M}}{f}^{\mathrm{F}, m_{1}, . ., m_{r}}(q)$ together with an additional marked oriented edge $\vec{e}$ that can be oriented in any possible oriented edge (including the $\vec{e}_{i}$ 's). We can compute the cardinality of $\tilde{\mathrm{M}}_{f}^{\mathrm{F}, m_{1}, . ., m_{r}}(q)$ in two ways. The first one is to use the definition, which gives the product between the amount of possible values of $\vec{e}$ and the cardinality of $\stackrel{\mathrm{M}}{f}_{f}^{\mathrm{F}, m_{1}, . ., m_{r}}(q)$, this gives the left-hand side of (9). The other one is to define a bijection between $\tilde{\mathrm{M}}_{f}^{\mathrm{F}, m_{1}, . ., m_{r}}(q)$ and $\mathrm{M}_{f}^{\mathrm{F}, \vec{c}}(q)$ with an ordering of its trees respecting that the $i$-th tree has size $m_{i}$ and then assigning one root edge to each tree. Therefore the cardinality of $\tilde{\mathrm{M}}_{f}^{\mathrm{F}, m_{1}, . ., m_{r}}(q)$ is obtained by the multiplication shown in the right-hand side of $(9)$, where $\prod_{k} c_{k}$ ! comes from the ordering needed only for the trees of each size.

Remark 19. It is important to remark that we can do this procedure as soon as we work with two types of rootings of the same family of maps. The justification is subtle since if one unroots a given map, certain symmetries may appear, breaking down the argument. Instead, when distinguishing more than one edge symmetries do not appear and this type of identities follow, since any automorphism of a map that fixes one oriented edge, fixes all of them.

\subsection{Counting relation between maps with a boundary and maps with a simple boundary}

The main interest of this section is to compute the generating functions of the maps with a simple boundary, as they appear in the bijection presented in Section 3.1. To do it, we are going to adapt the technique introduced in [BG09] that were used to link the generating function of quadrangulations with a boundary to that of quadrangulations with a simple boundary.

Let us start by noting that the set of maps with a simple boundary and $f$ faces is infinite. Instead, one needs to specify the number of edges and the size of the boundary.

We define the following generating functions

$$
\begin{aligned}
& \mathcal{B}(x, y)=\sum_{e=0}^{\infty} \sum_{p=0}^{\infty} b_{e, p} x^{e} y^{p}, \\
& \mathcal{S}(x, y)=\sum_{e=0}^{\infty} \sum_{p=0}^{\infty} s_{e, p} x^{e} y^{p}
\end{aligned}
$$

where $b_{e, p}$, resp. $s_{e, p}$, is the number of general maps with $e$ total edges and among which $p$ edges are on the boundary, where the boundary is simple for the case of $s_{e, p}$. 
Similarly to [BG09], we obtain the following identity relying $\mathcal{S}$ and $\mathcal{B}$.

$$
\mathcal{S}(x, y \mathcal{B}(x, y))=\mathcal{B}(x, y) .
$$

Let us sketch the justification: a rooted map with a general boundary $\mathfrak{m}^{b}$ can be decomposed for some $p \in \mathbb{N}$ into $\left(\mathfrak{s m}^{b},\left(\mathfrak{m}_{i}^{b}\right)_{i=1}^{p}\right)$ where:

- the map $\mathfrak{s m}^{b}$ is the maximal simple boundary connected component of the root-edge which has boundary size $p$, for some $p \in \mathbb{N}^{*}$.

- the map $\mathfrak{m}_{i}^{b}$ is a rooted map with general boundary equal to the map hanging from the $i$-th vertex in the boundary of $\mathfrak{s m}^{b}$ (numbered following the root edge) and rooted in the first edge in $\mathfrak{m}_{i}^{b}$ found when following the boundary of $\mathfrak{m}^{b}$. Beware this map can be reduced to a point, in such a case it is unrooted.

It turns out that this decomposition defines a bijection. Because of this, for each edge in the boundary of a general map with simple boundary we count a weight $y \mathcal{B}(x, y)$ to recover all maps with a boundary. The weight $y \mathcal{B}(x, y)$ is associated to the weight of the edge in the boundary and the map hanging from the tail of this edge consider as oriented following the contour of the simple boundary in the sense of the root-edge.

The only difference with [BG09], is that the external boundary may have any possible length. For the case of quadrangulations the external boundary has to be of even length; this forces in [BG09] the second coordinate in the left-hand side of eq. (11) to be $y W(x, y)^{2}$, with $W$ the generating function with coefficients $w_{e, p}$ counting the number of quadrangulations with $e$ edges and boundary of size $2 p$. When applying this technique, depending on the family of maps under study, it is important to take into account this type of restriction to obtain non-degenerate cases.

Now we use this change of variable to discover $\mathcal{S}$. To start, it is well known (see, for example, [FS09, VII.8.2]) that $\mathcal{B}(x, y)$ satisfies

$$
\mathcal{B}(x, y)=1+y^{2} x \mathcal{B}(x, y)^{2}+\frac{x y}{1-y}(\mathcal{B}(x, 1)-y \mathcal{B}(x, y))
$$

where $\mathcal{B}(x, 1)$ is the counting formula for general maps, with the following explicit form

$$
\mathcal{B}(x, 1)=\sum_{e=0}^{\infty} \frac{2 \cdot 3^{e}}{(e+1)(e+2)}\left(\begin{array}{c}
2 e \\
e
\end{array}\right) x^{e}=-\frac{1}{54 x^{2}}\left(1-18 x-(1-12 x)^{3 / 2}\right) .
$$

Now, turning into general maps with simple boundary, make the change of variable $z=$ $y \mathcal{B}(x, y)$ in eq. (12) to obtain

$$
\mathcal{S}(x, z)=1+x z^{2}+\frac{x z}{\mathcal{S}(x, z)-z}(\mathcal{B}(x, 1)-z) .
$$

This gives a quadratic equation for $\mathcal{S}$, obtaining the desired function $\mathcal{S}$ as the only possible solution of this equation with positive coefficients

$$
\mathcal{S}(x, z)=\frac{1}{2}\left(1+z+x z^{2}-\sqrt{\left(-x z^{2}+z+1\right)^{2}-\frac{2 z\left(1+36 x-(1-12 x)^{3 / 2}\right)}{27 x}}\right) .
$$


Its first coefficients are shown in the expansion $\mathcal{S}(x, z)=1+x z+2 x^{2} z+x z^{2}+9 x^{3} z+$ $x^{2} z^{2}+54 x^{4} z+5 x^{3} z^{2}+378 x^{5} z+32 x^{4} z+x^{3} z^{3}+\cdots$.

This generating function codes the number of general maps with a simple boundary. The coefficients may be recovered from it by doing a sequence of derivations. Nevertheless, they do not have a closed form to our knowledge.

\subsection{Counting results}

Before presenting the results, let us start by recalling that the number of (rooted) trees with $n$ edges are counted by Catalan numbers $\left\{\mathcal{C}_{n}\right\}_{n \in \mathbb{N}}$ (see, for example, Section 1 in [Ber07])

$$
\left|\mathrm{T}_{n}\right|=\mathcal{C}_{n}:=\frac{1}{n+1}\left(\begin{array}{c}
2 n \\
n
\end{array}\right)
$$

\subsubsection{Tree and forest decorated maps}

Let us now obtain the formulas that directly come from the bijection for tree-decorated maps. The counting formulas obtained for maps with multiple simple boundaries come from [Kri07] for the case of triangulations and from [BF18] in the case of quadrangulations.

Corollary 20. Set $m=\left(m_{1}+2 m_{2}\right) / 2$. The number of tree-decorated triangulations with boundary of size $m_{1} \geqslant 1$ and a tree of size $m_{2}$ is

$$
\left|\mathrm{B}_{f, m_{1}}^{\mathrm{T}, m_{2}}(3)\right|=2^{f-2 m} \frac{(3 f / 2+m-2) ! !}{(f / 2-m+1) !(f / 2+3 m) ! !} \frac{2 m}{m_{2}+1}\left(\begin{array}{c}
4 m \\
2 m
\end{array}\right)\left(\begin{array}{c}
2 m_{2} \\
m_{2}
\end{array}\right),
$$

where $n$ !! stands for double factorial (or semifactorial). The number of tree-decorated quadrangulations with boundary of size $m_{1} \geqslant 1$ and a tree of size $m_{2}$ is

$$
\left|\mathrm{B}_{f, m_{1}}^{\top, m_{2}}(4)\right|=3^{f-m} \frac{(2 f+m-1) !}{(f+2 m) !(f-m+1) !} \frac{2 m}{m_{2}+1}\left(\begin{array}{c}
3 m \\
m
\end{array}\right)\left(\begin{array}{c}
2 m_{2} \\
m_{2}
\end{array}\right) .
$$

Proof. This is obtained from Proposition 14 and Lemma 9 adapted to tree-decorated maps with a simple boundary. The formula for the number of trees with $m_{2}$ edges are Theorem 1 of [Kri07] and Section 2.2 of [BG09] respectively.

We can now discuss the results for forests.

Corollary 21. The cardinal of r-forest decorated triangulations, with trees of prescribed sizes $m_{1}, m_{2}, \ldots, m_{r}$ and $m:=\sum m_{i}$ and $m+r \geqslant f / 2+2$ is given by

$$
\left|\mathrm{M}_{f}^{\mathrm{F}, \vec{c}}(3)\right|=2^{f-2 m} \frac{3 f}{\prod_{i \in \mathbb{N}} c_{k} !} \frac{(3 f / 2+m-2) ! !}{(f / 2-m+2-r) !(f / 2+3 m) ! !} \prod_{i=1}^{r} \frac{1}{m_{i}+1}\left(\begin{array}{c}
4 m_{i} \\
2 m_{i}, m_{i}, m_{i}
\end{array}\right)
$$


where $\vec{c}$ is defined as in eq. (10). and r-forest decorated quadrangulations, with trees of size $m_{1}, m_{2}, \ldots, m_{r}$, and $m+r \geqslant f+2$ is given by:

$$
\left|\mathbf{M}_{f}^{\mathrm{F}, \vec{c}}(4)\right|=3^{f-m} \frac{4 f}{\prod_{i \in \mathbb{N}} c_{k} !} \frac{(2 f+m-1) !}{(f+2 m) !(f-m+2-r) !} \prod_{i=1}^{r} \frac{1}{m_{i}+1}\left(\begin{array}{c}
3 m_{i} \\
m_{i}, m_{i}, m_{i}
\end{array}\right) .
$$

where $\vec{c}$ is defined as in eq. (10).

Proof. This is obtained from Proposition 14, Lemma 9 adapted to forest-decorated maps with a simple boundary, the formula for the number of trees with $m_{i}$ edges and Theorem 1 of [Kri07] and Theorem 1.2 of [BF18] respectively, giving that

$$
\left|\stackrel{\mathrm{M}}{f}_{f}^{\mathrm{F}, m_{1}, . ., m_{r}}(3)\right|=\frac{2^{f-2 m}(3 f / 2+m-2) ! !}{(f / 2-m+2-r) !(f / 2+3 m) ! !} \prod_{i=1}^{r} \frac{2 m_{i}}{m_{i}+1}\left(\begin{array}{c}
4 m_{i} \\
2 m_{i}, m_{i}, m_{i}
\end{array}\right)
$$

and

$$
\left|\stackrel{\circ}{\mathrm{M}}_{f}^{\mathrm{F}, m_{1}, . ., m_{r}}(4)\right|=3^{f-m} \frac{(2 f+m-1) !}{(f+2 m) !(f-m+2-r) !} \prod_{i=1}^{r} \frac{2 m_{i}}{m_{i}+1}\left(\begin{array}{c}
3 m_{i} \\
m_{i}, m_{i}, m_{i}
\end{array}\right) .
$$

We conclude using the re-rooting procedure condensed in (9).

Remark 22. Let us note that Corollary 2 can be obtained from Corollary 21 using $r=1$.

Similar counting formulas can be obtained for triangulations of girth bigger than 2 (loopless triangulations) and 3 (triangulations without multiple edges); and simple quadrangulations (see [BF18]). In Corollary 21, one could also consider a generalization for "tree-decorated maps with boundaries" an analog of the tree-decorated maps with a boundary for multiples boundaries. We hope that the interested reader will have no problem in finding the formula.

As discussed in Section 4.2, the number of general maps with a simple boundary does not have a closed formula to our knowledge, still it is possible to obtain the number of general maps decorated by a tree once extracted the coefficients $s_{e, p}$ with $p$ even. More formally, the number of general maps decorated by a tree of size $m$ and with $e$ edges is given by $\mathcal{C}_{m} s_{e+m, 2 m}$, which is justified by Theorem 4 .

The cardinal of bubble maps can be obtained from Proposition 18.

Corollary 23. The cardinal of non-crossing circuit decorated bubble-maps $(\mathfrak{m}, \mathfrak{c})$ with $e+m$ edges decorated by a circuit of size $2 m$ and with root in an oriented edge of the map $\mathfrak{m}$ is

$$
\left|\mathbf{M}_{E=e+m}^{\mathrm{C}, m}\right|=3^{e} \frac{(2 e+2 m-1) !}{e !(e+2 m+1) !} \frac{2(e+m)}{m+1}\left(\begin{array}{c}
4 m \\
2 m, m, m
\end{array}\right) .
$$

Proof. This is obtained from Proposition 18, the formula for the number of trees with $m$ edges, the results of [FG15, Section 2.2] together with [BG09, Section 2.2] and a type of re-rooting procedure, similar to the one in Section 4.1.

We leave to the reader the computation of the formula of maps decorated by a special type of trees, as for example trees with prescribed degree distribution, see for example [Sta99]. 


\subsubsection{Spanning tree-decorated maps}

In this subsection, we discuss the consequence of our result for spanning tree-decorated maps. In this case, the counting formula of spanning tree-decorated maps for general face degrees was given by Mullin [Mul67], where the root does not necessarily belongs to the tree. It states that spanning tree-decorated maps with $e$ edges are in correspondence with a pair of trees with $e$ and $e+1$ edges, i.e. is counted by $\mathcal{C}_{e} \mathcal{C}_{e+1}$. Later Bernardi [LW72, CDV86, Ber07] gave a bijective proof of this result.

We denote by $\dot{M}_{f}^{S T}$, resp. $\mathrm{M}_{f}^{\mathrm{ST}}$, the set of spanning tree-decorated maps on $f$ faces with one root-edge in the tree, resp. in any possible oriented edge of the map. Note that in the case of triangulations we obtain that for $f$ faces the number of vertices is $2+f / 2$ and the number of edges is $3 / 2 f$, therefore, the number of spanning-tree decorated triangulations is

$$
\left|\mathrm{M}_{f}^{\mathrm{ST}}(3)\right|=\left|\mathrm{M}_{f}^{\mathrm{T}, f / 2+1}(3)\right|=\frac{12 f}{(f+4)(f+2)^{2}}\left(\begin{array}{c}
2 f \\
f, f / 2, f / 2
\end{array}\right) .
$$

In the case of quadrangulations, the condition of having $f$ faces implies that it has $f+$ 2 vertices and $2 f$ edges. Therefore, we obtain the counting formula for spanning-tree decorated quadrangulations.

$$
\begin{gathered}
\left|\stackrel{\mathrm{M}}{f}_{f}^{\mathrm{ST}}(4)\right|=\left|\stackrel{\mathrm{M}}{f}^{\mathrm{T}, f+1}(4)\right|=\frac{2}{(f+1)(f+2)}\left(\begin{array}{c}
3 f \\
f, f, f
\end{array}\right) \\
\left|\mathrm{M}_{f}^{\mathrm{S \top}}(4)\right|=\left|\mathrm{M}_{f}^{\mathrm{T}, f+1}(4)\right|=\frac{4 f}{(f+1)^{2}(f+2)}\left(\begin{array}{c}
3 f \\
f, f, f
\end{array}\right) .
\end{gathered}
$$

Here we recover the results ontained from the Lehman and Walsh's Bijection [LW72]. In fact, the dual of $\stackrel{\mathrm{M}}{f}_{f}^{\mathrm{ST}}(3)$ are the spanning tree-decorated 3-regular maps with $f$ vertices, and that of $\dot{M}_{f}^{\mathrm{ST}}(4)$ are the spanning tree-decorated 4-regular maps with $f$ vertices. These can be counted as appears in Section 6.2 of [BM11], where they explicit the formula for more general families of maps, given that (dual) trees with prescribed degree distribution are easily counted.

\subsubsection{Spanning r-forest decorated maps}

Corollary 24. The cardinality of spanning $r$-forest-decorated triangulations, with trees of size $m_{1}, m_{2}, \ldots, m_{r}$ and $m:=\sum m_{i}$, is given by

$$
\left|\mathrm{M}_{f}^{\mathrm{SF}, \vec{c}}(3)\right|=4^{r-2} \frac{3 f}{\prod_{k \in \mathbb{N}} c_{k} !} \frac{(2 f+2-r) ! !}{(2 f+6-3 r) ! !} \prod_{i=1}^{r} \frac{1}{m_{i}+1}\left(\begin{array}{c}
4 m_{i} \\
2 m_{i}, m_{i}, m_{i}
\end{array}\right),
$$

where $\vec{c}$ is defined as in eq. (10).

The cardinality of spanning $r$-forest-decorated quadrangulations, with trees of size $m_{1}, m_{2}, \ldots, m_{r}$, is given by:

$$
\left|\mathrm{M}_{f}^{\mathrm{SF}, \vec{c}}(4)\right|=3^{r-2} \frac{4 f}{\prod_{k \in \mathbb{N}} c_{k} !} \frac{(3 f-r+1) !}{(3 f-2 r+4) !} \prod_{i=1}^{r} \frac{1}{m_{i}+1}\left(\begin{array}{c}
3 m_{i} \\
m_{i}, m_{i}, m_{i}
\end{array}\right),
$$


where $\vec{c}$ is defined as in eq. (10).

Proof. A triangulation with $f$ faces has $2+f / 2$ vertices, which has to be equal to $m+r=$ $\sum_{i=1}^{r} m_{i}+r$, the number of vertices covered by the forest. As before, a quadrangulation with $f$ faces has $f+2$ vertices, which has to be equal to $m+r$. The result follows from Corollary 21.

Remark 25. From this formula it is also possible to deduce Corollary 3.

Remark 26. Notice that we kept the expressions for these spanning-tree decorated quadrangulations, with root in the tree and in any possible oriented edge of the map. We want to point out that the right-hand side of (24) looks like a possible generalization of the Catalan numbers. More rigorously, for $n, m \in \mathbb{N}, m \geqslant 1$ define:

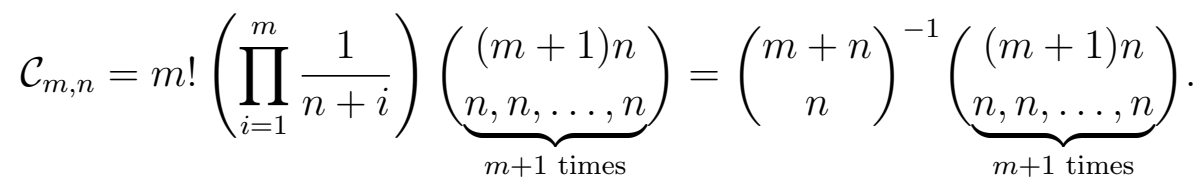

When $m=1$, we recover the Catalan numbers and, for this definition, $\mathcal{C}_{2, f}$ counts $\left|\mathrm{M}_{f}^{\mathrm{ST}}(4)\right|$. To our knowledge, this extension has not been defined so far and it does not appear in the OEIS ${ }^{4}$.

From the definition it is not clear to see that $\mathcal{C}_{m, n}$ is, in fact, an integer. Luckily for us, Vincent Jugé found an elegant, analytical proof of this fact that we present in the following proposition.

Proposition 27. For all $n, m \in \mathbb{N}$ and $m \geqslant 1, \mathcal{C}_{m, n}$ is an integer.

Proof. Define $\nu_{p}(k)$ as the largest power of $p$ prime that divides $k \in \mathbb{N}$. Recall that by Legendre's formula

$$
\nu_{p}(k !)=\sum_{i=1}^{\infty}\left\lfloor\frac{k}{p^{i}}\right\rfloor .
$$

Thanks to this, we can calculate the maximal power of $p$ prime that divides $\mathcal{C}_{m, n}$

$$
\begin{aligned}
& \nu_{p}((m+1) n !)-\nu_{p}\left((n !)^{m+1}\right)-\nu_{p}((n+m) !)+\nu_{p}(n !)+\nu_{p}(m !) \\
& =\sum_{i=1}^{\infty} \underbrace{\left\lfloor\frac{(m+1) n}{p^{i}}\right\rfloor-(m+1)\left\lfloor\frac{n}{p^{i}}\right\rfloor}_{=:(1) \geqslant 0} \underbrace{-\left\lfloor\frac{m+n}{p^{i}}\right\rfloor+\left\lfloor\frac{n}{p^{i}}\right\rfloor+\left\lfloor\frac{m}{p^{i}}\right\rfloor}_{=:(2) \geqslant-\lfloor}
\end{aligned}
$$

Note that to conclude we just need to show that each term in the summation is bigger than or equal to zero. To do this notice that we just need to show that it cannot happen simultaneously $(1)=0$ and $(2)=-1$.

\footnotetext{
${ }^{4}$ March,4, 2019 update: it has been added for $m=2,3$.
} 
Assume that this is the case and write

$$
\begin{aligned}
& n=k p^{i}+l_{n}, \\
& m=k^{\prime} p^{i}+l_{m},
\end{aligned}
$$

with $0 \leqslant l_{n}, l_{m}<p^{i}$ and $l_{m}<m$. Note that the fact that $(1)=0$ implies that

$$
l_{n}(m+1)<p^{i} .
$$

Futhermore, the fact that $(2)=-1$ implies that $l_{n} \neq 0$ and

$$
l_{n}+l_{m} \geqslant p^{i} .
$$

Together with (28) this implies that $m l_{n}<l_{m} \leqslant m$ which implies that $l_{n}=0$ so we have a contradiction.

\section{Acknowledgments}

We would like to thank Jérémie Bettinelli, Mireille Bousquet-Mélou, Éric Fusy, Vincent Jugé, Jean-François Marckert, Grégory Miermont and Neil Sloane for interesting and fruitful discussions. The work of this paper started and was partially done during various trips of both authors financed by ERC grant LiKo 676999. Also, we would like to thank Núcleo Milenio Stochastic models of complex and disordered system for inviting us to Chile, where part of this work was done, and to "Mobilité junior" of LaBRI who financed part of the trip of L.F. The research of L.F. has been partially supported by ANR GRAAL (ANR-14-CE25-0014). The research of A.S. is supported by ERC grant LiKo 676999.

\section{References}

[BBG11] Gaetan Borot, Jérémie Bouttier, and Emmanuel Guitter. A recursive approach to the $\mathrm{O}(\mathrm{n})$ model on random maps via nested loops. Journal of Physics A: Mathematical and Theoretical, 45(4):045002, 2011.

[BDFG04] Jérémie Bouttier, Philippe Di Francesco, and Emmanuel Guitter. Planar maps as labeled mobiles. The Electronic Journal of Combinatorics, 11(1):\#R69, 2004.

[Ber07] Olivier Bernardi. Bijective counting of tree-rooted maps and shuffles of parenthesis systems. The ElectronicJCournal of Combinatorics, 14(1):\#R9, 2007.

[Bet15] Jérémie Bettinelli. Scaling limit of random planar quadrangulations with a boundary. 51(2):432-477, 2015.

[BF18] Olivier Bernardi and Éric Fusy. Bijections for planar maps with boundaries. Journal of Combinatorial Theory, Series A, 158:176-227, 2018.

[BG09] Jérémie Bouttier and Emmanuel Guitter. Distance statistics in quadrangulations with a boundary, or with a self-avoiding loop. Journal of Physics A: Mathematical and Theoretical, 42(46):465208, 2009. 
[BM11] Mireille Bousquet-Mélou. Counting planar maps, coloured or uncoloured. In 23rd British Combinatorial Conference, volume 392, pages 1-50. 2011.

[BM17] Jérémie Bettinelli and Grégory Miermont. Compact brownian surfaces I: Brownian disks. Probability Theory and Related Fields, 167(3-4):555-614, 2017.

[CC16] Alessandra Caraceni and Nicolas Curien. Self-avoiding walks on the UIPQ. arXiv:1609.00245, 2016.

[CDV86] Robert Cori, Serge Dulucq, and Gérard Viennot. Shuffle of parenthesis systems and baxter permutations. Journal of Combinatorial Theory, Series A, 43(1):1$22,1986$.

[DG18] Jian Ding and Ewain Gwynne. The fractal dimension of Liouville quantum gravity: universality, monotonicity, and bounds. arXiv:1807.01072, 2018.

[DK88] Bertrand Duplantier and Ivan Kostov. Conformal spectra of polymers on a random surface. Physical review letters, 61(13):1433, 1988.

[Edm60] Jack Edmonds. A combinatorial representation of polyhedral surfaces. Notices of the American Mathematical Society, 7, 1960.

[FG15] Éric Fusy and Emmanuel Guitter. Comparing two statistical ensembles of quadrangulations: a continued fraction approach. arXiv:1507.04538, 2015.

[FS09] Philippe Flajolet and Robert Sedgewick. Analytic combinatorics. Cambridge University Press, Cambridge, 2009.

[FS19] Luis Fredes and Avelio Sepúlveda. Limits of tree-decorated maps. To appear, 2019.

[GHS17] Ewain Gwynne, Nina Holden, and Xin Sun. A mating-of-trees approach to graph distances in random planar maps. arXiv:1711.00723, 2017.

[GJ04] Ian Goulden and David Jackson. Combinatorial enumeration. Courier Corporation, 2004.

[GM16] Ewain Gwynne and Jason Miller. Convergence of the self-avoiding walk on random quadrangulations to $\mathrm{SLE}_{8 / 3}$ on $\sqrt{8 / 3}$-liouville quantum gravity. arXiv:1608.00956, 2016.

[GM19] Ewain Gwynne and Jason Miller. Convergence of the free Boltzmann quadrangulation with simple boundary to the brownian disk. To appear in Annales de l'institut Henri Poincaré, 2019.

[JS04] Wolfhard Janke and Adriaan Schakel. Geometrical vs. fortuin-kasteleyn clusters in the two-dimensional q-state potts model. Nuclear Physics B, 700(13):385-406, 2004.

[Kri07] Maxim Krikun. Explicit enumeration of triangulations with multiple boundaries. The Electronic Journal of Combinatorics, 14(1):\#R61, 2007.

[LG05] Jean-François Le Gall. Random trees and applications. Probability surveys, $2: 245-311,2005$. 
[LG13] Jean-François Le Gall. Uniqueness and universality of the brownian map. The Annals of Probability, 41(4):2880-2960, 2013.

[LW72] Alfred Lehman and Timothy Walsh. Counting rooted maps by genus ii. Journal of Combinatorial Theory, Series B, 13(2):122-141, 1972.

[Mie13] Grégory Miermont. The brownian map is the scaling limit of uniform random plane quadrangulations. Acta mathematica, 210(2):319-401, 2013.

[MM06] Jean-François Marckert and Abdelkader Mokkadem. Limit of normalized quadrangulations: the brownian map. The Annals of Probability, 34(6):2144-2202, 2006.

[MS16] Jason Miller and Scott Sheffield. Quantum Loewner evolution. Duke Mathematical Journal, 165(17):3241-3378, 2016.

[Mul67] Ronald Mullin. On the enumeration of tree-rooted maps. Canadian Journal of Mathematics, 19:174-183, 1967.

[Pom13] Christian Pommerenke. Boundary behaviour of conformal maps, volume 299. Springer Science \& Business Media, 2013.

[Sch98] Gilles Schaeffer. Conjugaison d'arbres et cartes combinatoires aléatoires. PhD thesis, Université Bordeaux 1, 1998.

[She16] Scott Sheffield. Quantum gravity and inventory accumulation. The Annals of Probability, 44(6):3804-3848, 2016.

[Sta99] Richard P. Stanley. Enumerative combinatorics. Vol. 2, volume 62 of Cambridge Studies in Advanced Mathematics. Cambridge University Press, Cambridge, 1999. With a foreword by Gian-Carlo Rota and appendix 1 by Sergey Fomin.

[Tut62] William Tutte. A new branch of enumerative graph theory. Bulletin of the American Mathematical Society, 68(5):500-504, 1962. 